\title{
THE EXISTENCE OF ANORMAL CHAINS
}

\section{DAVID BLACKWELL}

1. Introduction. Let $\bar{B}$ be a Borel field of subsets of a space $X$, and let $P(x, E)$ be for fixed $x$ a probability measure on $\bar{B}$ and for fixed $E$ a $\bar{B}$-measurable function of $x . P(x, E)$ may be considered as representing the transition probability of going from $x$ into $E$ in a single trial. Denote by $\Omega$ the space of sequences $\omega:\left(x_{0}, x_{1}, \cdots\right)$ where $x_{i} \in X$ and by $\bar{E}$ the Borel field of subsets of $\Omega$ determined by all sets

$$
\left\{x_{i} \in E\right\} \text {, where } E \in \bar{B}, \quad i=1,2, \cdots \text {. }
$$

Doob [2, pp. 102-103 $]^{1}$ has shown that there exists for each $x \in X$ a probability measure $P_{x}(S)$ defined on $\bar{E}$ such that for every $P_{x}$-integrable function $f\left(x_{1}, \cdots, x_{n}\right)$

(1) $\int f(\omega) d P_{x}=\iint \cdots \int f\left(x_{1}, \cdots, x_{n}\right) d P\left(x_{n-1}, x_{n}\right) \cdots d P\left(x, x_{1}\right)$,

that $\Omega$ with the measure $P_{x}$ is a Markoff process, that is, $E\left(x_{1}, \cdots, x_{n} ; g\right)$ $=E\left(x_{n} ; g\right)$ where $g=g\left(x_{n+1}, x_{n+2}, \cdots\right)$ and the $E$ 's denote conditional expectations with respect to the indicated variables, and that $E\left(x_{1}, \cdots, x_{r} ; f\right)$ is the function obtained by carrying out the first $n-r$ integrations in (1).

Write $Q(x, E)=P_{x}\left(\lim \sup \left\{x_{i} \in E\right\}\right)$, so that $Q(x, E)$ represents the probability of entering $E$ infinitely often, starting from $x$. Following Doblin $[1$, p. 68 et seq.] we make the following definitions for sets of $\bar{B}: E$ is inessential if $Q(x, E)=0$ for all $x$, and essential otherwise. An essential set is improperly essential if it is a denumerable sum of inessential sets, and absolutely essential otherwise. A finite or denumerable sum of improperly essential sets is consequently improperly esessential. $E$ is closed if $P(x, E)=1$ for all $x \in E$, and a closed set is indecomposable if it does not contain two disjunct non-empty closed subsets. An absolutely essential indecomposable set is said to be nor$m a l$ if it contains a closed set which contains no improperly essential subsets and anormal otherwise. If $X$ is a normal set, we shall say that the Markoff chain determined by $P(x, E)$ is a normal chain.

Doblin [1] has obtained for normal chains many elegant results which are considerably more complicated for the anormal case. For example $[1$, p. 81$]$ in the normal case there exists a closed set $G$ such

Received by the editors September 16, 1944.

1 Numbers in brackets refer to the references cited at the end of the paper. 
that $Q(x, E)=1$ for every essential subset $E$ of $G$ and every $x \in G$; in the anormal case $G$ can no longer be chosen independently of $E$. It is consequently of interest to investigate conditions for normality. Doblin $[1$, p. 82] has given a quite general sufficient condition for the occurrence of the normal case, and no example of an anormal chain has hitherto been given. The purpose of this paper is to give a simple necessary and sufficient condition for the occurrence of the normal case, consisting merely in the measurability of the function $f(x)$ which represents the probability that, starting from $x$, the point remains indefinitely in an improperly essential set. An example of an anormal chain is also given.

2. The normal case. We restate a result of Doblin $[1$, p. 80$]$ in the following theorem.

THEOREM 1. If $X$ is indecomposable and absolutely essential, the closed set $\{Q(x, E)=1\}$ is non-empty if and only if $E$ is absolutely essential.

The following lemma asserts that the probability of entering $E$ infinitely often, starting from $y$ at the $j$ th trial, is independent of $j$ :

Lemma. With respect to any $P_{x}$ measure,

$$
P_{x}\left(x_{j}=y ; \lim \sup \left\{x_{i} \in E\right\}\right)=Q(y, E) .
$$

Proof. If $f_{m, n}$ is the characteristic function of the set $E(m, n)$ $=\sum_{m \leqq i \leqq n}\left\{x_{i} \in E\right\}$, it follows from (1) that

$$
\begin{aligned}
P_{y}(E(m, n)) & =\iint \cdots \int f_{m, n} d P\left(x_{n-1}, x_{n}\right) \cdots d P\left(y, x_{1}\right) \\
& =P_{x}\left(x_{j}=y ; E(m+j, n+j)\right) .
\end{aligned}
$$

Letting first $n$, then $m$, become infinite, we obtain (2).

The following theorem, which is new and of some independent interest, asserts that for any two sets $T$ and $E$, unless there are points of $T$ which are practically certain to enter $E$ infinitely often, it is impossible for a point to enter both $T$ and $E$ infinitely of ten.

Theorem 2. If $Q(x, E) \leqq a<1$ for all $x \in T$, then for all $x$

$$
h(x)=P_{x}\left(\lim \sup \left\{x_{i} \in E\right\} \cdot \lim \sup \left\{x_{i} \in T\right\}\right)=0 .
$$

Proof. Define $E(N, n)=\left\{x_{i} \notin E\right.$ for $\left.N \leqq i<n, x_{n} \in E\right\}, T(N, n)$ $=\left\{x_{i} \notin T\right.$ for $\left.N \leqq i<n, x_{n} \in T\right\}$. Now for fixed $N$,

$$
h(x) \leqq \sum_{N<n<r} P_{x}\left(E(N, n) T(n, r) \lim \sup \left\{x_{i} \in E\right\}\right) .
$$


But

$$
\begin{aligned}
P_{x}\left(E(N, n) T(n, r) \lim \sup \left\{x_{i} \in E\right\}\right) \\
=\int_{E(N, n) T(n, r)} P_{x}\left(x_{1}, \cdots, x_{r} ; \lim \sup \left\{x_{i} \in E\right\} d P_{x}\right. \\
=\int_{E(N, n) T(n, r)} Q\left(x_{r}, E\right) d P_{x} \leqq a P_{x}(E(N, n) T(n, r),
\end{aligned}
$$

by the lemma and the fact that $x_{r}$ belongs to $T$ in the domain of integration. Using the last inequality in (4), we obtain

$$
h(x) \leqq a \sum_{N<n<r} P_{x}(E(N, n) T(n, r))=a P_{x}\left(\sum_{N<n<r} E(N, n) T(n, r)\right) .
$$

Letting $N$ become infinite we obtain $h(x) \leqq a h(x)$, which implies (3). Our necessary and sufficient condition for the occurrence of the normal case, given in Theorem 3 , is an easy consequence of Theorems 1 and 2 .

Theorem 3. Let $X$ be absolutely essential and indecomposable. Then $X$ is normal if and only if $f(x)=1$.u.b.imp.ess. $E Q(x, E)$ is $\bar{B}$-measurable.

Proof. By Theorem 1, for every improperly essential $E$ and every $x$ we have $Q(x, E)<1$. It follows that $f(x)<1$ for all $x$, since a denumerable sum of improperly essential sets is improperly essential. If $f(x)$ is measurable, there exists an $a<1$ such that $T=\{f(x)<a\}$ is absolutely essential; for $X=\sum_{n=1}^{\infty}\{f(x)<1-1 / n\}$, and not all these sets can be improperly essential. Denoting by $S$ the closed set $\{Q(x, T)$ $=1\}$, it follows from Theorem 1 that $S$ is non-empty and from Theorem 2 that $Q(x, E)=0$ for all $x \in S$ and all improperly essential $E$. In particular if $E \subset S$ and $E$ is not absolutely essential, $Q(x, E)=0$ for all $x \in E$, which by Theorem 2 in the special case $T=E$ implies that $E$ is inessential. Thus $S$ is a non-empty closed set containing no improperly essential subsets, and $X$ is normal.

Conversely if there exists such a subset $S$ it is easily verified that $f(x)=Q(x, X-S)$ and is therefore measurable.

3. An anormal chain. The space $X$ is the semi-infinite interval $0 \leqq x<\infty$, and $\bar{B}$ is the Borel field of all finite or denumerable subsets of $X$ and their complements. Let $a_{n}$ be any sequence of numbers such that $0<a_{n}<1, \prod_{0}^{\infty} a_{n}>0$. We define

$$
P(x, E)=a_{n} f(x+1, E)+\left(1-a_{n}\right) d(E),
$$


where $n$ is the largest positive integer not exceeding $x, d(E)$ is 1 if $E$ is non-denumerable and 0 otherwise, and $f(x, E)$ is the characteristic function of $E . P(x, E)$ is clearly a probability measure on $\bar{B}$ for fixed $x$. To verify that $P(x, E)$ is $\bar{B}$-measurable for fixed $E$, we may assume that $E$ is at most denumerable, since $P(x, C E)=1-P(x, E)$. For this case $P(x, E)=0$ except on an at most denumerable set and is consequently measurable. Since the probability of going from $x$ into $x+1$ is $a_{n}$ for $n \leqq x<n+1$ and since $\prod_{0}^{\infty} a_{n}>0$, every set containing all points $x+n, n=1,2, \cdots$, for some $x$ is essential. The closed sets are those nondenumerable sets which contain with $x$ all points $x+n$, so that $X$ is indecomposable. Finally $X$ is absolutely essential, since $\sum S_{n}=X$ implies that some $S_{n}$ is nondenumerable and hence esssential. Thus $X$ is anormal.

\section{REFERENCES}

1. Doblin, W. Chaines simples constantes de Markoff, Ann. Ecole Norm. vol. 3 (1940).

2. Doob, J. L., Stochastic processes with an integral-valued parameter, Trans. Amer. Math. Soc. vol. 44 (1938).

HOWARD UNIVERSITY 